\title{
Effect of Processing Conditions on Starch/GS Composite
}

\author{
Yu-jun Zhou ${ }^{1, a}$ \\ ${ }^{1}$ College of Chemistry and Environmental Engineering, Jiangxi Province Engineering Research \\ Center of Ecological Chemical Industry, Jiujiang University, Qianjin Eath Road No.551, Jiujiang City, \\ Jiangxi Province, People's Republic of China \\ a82294546@qq.com
}

Keywords: Composite, Hot-pressing, Renewable resource, Performance.

\begin{abstract}
Starch/GS composite was prepared by hot-pressing method. The effects of processing conditions including hot-pressing time and amount of mixing medium on the performance of composites were studied. Results showed that tensile strength first increased and then decreased with hot-pressing time. When hot-pressing time was $10 \mathrm{~min}$, composite achieved maximum tensile strength, $2.14 \mathrm{MPa}$, which was $20.9 \%$ higher than that of composite prepared for $14 \mathrm{~min}$. Strength at tensile fracture and tensile elongation showed similar changing law with hot-pressing time as tensile strength. When hot-pressing time was $10 \mathrm{~min}$, strength at tensile fracture composite was $1.16 \mathrm{MPa}$ and tensile elongation was $12.61 \%$. Amount of mixing medium had obvious effect on strength of composite. Too much or too less water both decreased tensile strength and strength at tensile fracture. When water amount was $10 \mathrm{~mL}$, tensile strength and strength at tensile fracture both reached their maximum values and that were 3.01 $\mathrm{MPa}$ and 2.83 MPa respectively.
\end{abstract}

\section{Introduction}

Developing plant based composite, instead of wood and plastics, is of great importance to decrease excessive deforestation for protecting ecological environment and relieve white pollution resulted from wide application of plastics. Plant is a kind of important renewable natural resource. Utilizing plant in the preparation of composite is also conductive to ease resources crisis. Environmentally friendly materials prepared with plant as raw materials have a wide range of uses, for example, preparing packaging materials, furniture and toys. When plant based composite is made, straw of crops is the most often used. Kurokochi et al ${ }^{[1]}$ manufactured binderless boards from rice straw by hot-pressing in order to evaluate the morphological effect of particles from rice straw. For the morphological effect, they examined the influence of particle size and morphological parts of rice straw, respectively. Internal bonding (IB), thickness swelling (TS) and water absorption (WA) were measured as the properties of the boards. As a result, binderless boards were successfully manufactured from rice straw powder smaller than $1 \mathrm{~mm}$. Regarding the effect of morphological part from rice straw, IB of the board made from stem powder was the highest, subsequently followed by leaf blade powder, and leaf sheath powder. IB tended to increase with decreasing ash and silica contents. Before the above mentioned study, a lot of others were performed ${ }^{[2-7]}$.

As crop straw, grass is also a kind of widely distributed plant resource with huge yield. Grass bears good strength and is adapt to prepare environmentally friendly composite. Here grass based composite was fabricated by hot-pressing method and effect of processing conditions including hot-pressing time and amount of mixing medium on the performances of composite was studied. When composite was fabricated, starch was used as adhesive matter. Starch is of natural origin. Using starch as raw material to fabricate composite is beneficial to increase the environmental compatibility of composite.

\section{Materials and Methods}

Materials. Grass was obtained from Jiujiang University's lawn and was dried in the sun before application. Grass was cut into about $1.5 \mathrm{~cm}$ long segments and then was sheared. After removing 
grass powder by to prepare grass slice, abbreviated as GS. Water was used as mixing medium and was obtained from urban water-supplying system. Dimethicone oil was purchased from Xilong chemical company (Shantou, China) and was used as received.

Preparation of Starch/GS Composite. A series of GS were weighed into beakers and then water was added, followed by stirring for 5 min so that GS was uniformly moistened. When the effect of hot-pressing time was studied, the amount of GS was $18 \mathrm{~g}$. And when the effect of mixing medium was studied, $15 \mathrm{~g}$ GS was used. Thereafter, starch was weighed and added into moistened rice straw, followed by stirring for $5 \mathrm{~min}$. The total mass of GS and starch was kept as $30 \mathrm{~g}$. A $125 \mathrm{~mm} \times 125 \mathrm{~mm}$ $\times 2 \mathrm{~mm}$ iron mold was prepared by brushing a layer of dimethicone oil onto the top and bottom surfaces of mold. Then the mixed raw material was put in the lower mold, upper mold plate was put on the lower mold and then was hot-pressed on MZ-3012 machine at 10MPa pressure. The mold was taken from hot-pressing machine. After cooling to room temperature, the product was taken out to be tested.

Measurement of Composite. Measurements of tensile performances and hardness were carried out as previously reported ${ }^{[2]}$.

\section{Results and Discussion}

The Effect of Hot-pressing Time on the Performance of Composite Materials. A series of Starch/GS composites containing 18g GS were prepared at different hot-pressing time. The change of tensile strength with the increase of hot-pressing time was drawn in Fig. 1. It can be seen that tensile strength first increases and then decreases with hot-pressing time. When hot-pressing time was 10 min, composite achieved maximum tensile strength, 2.14MPa. In Starch/GS composites, GS can not bind each other but is bond by starch. The binding between GS by starch needs a course. When time was short, GS did not obtain complete binding. But if hot-pressing time was too long, starch and GS would undergo structural change under the conditions of water and heat. Structural change resulted in novel structural defects and decreased strength of composite.

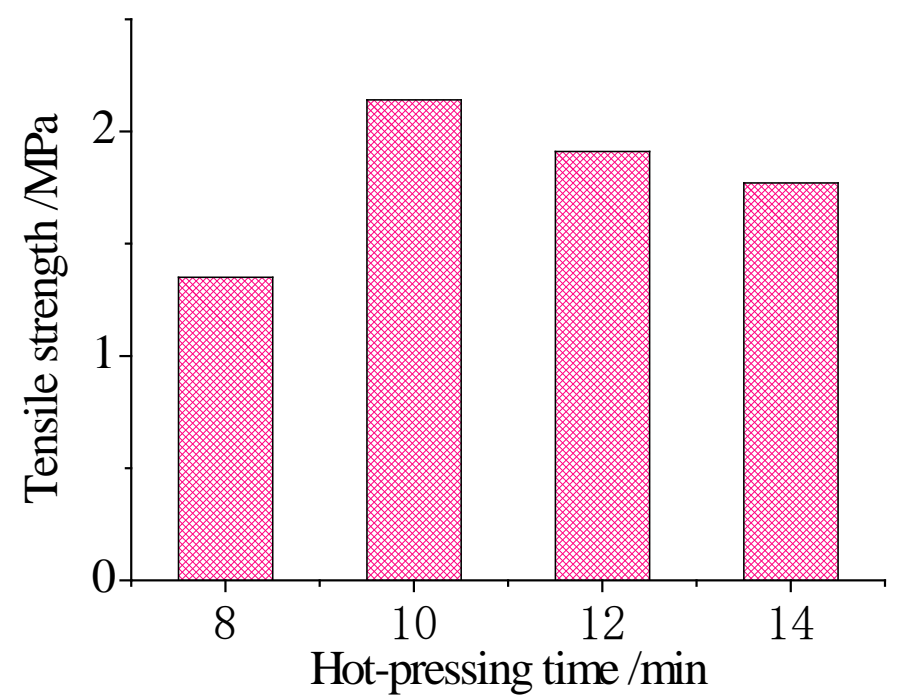

Fig. 1 The tensile strength of composites at different hot-pressing time.

The change of strength at tensile fracture with the increase of hot-pressing time was drawn in Fig. 2. It can be seen that as tensile strength, strength at tensile fracture first increases and then decreases with hot-pressing time. When hot-pressing time was $10 \mathrm{~min}$, composite achieved maximum strength at tensile fracture, $1.16 \mathrm{MPa}$. The change law of strength at tensile fracture resulted from similar cause as tensile strength. By comparing Fig. 1 with Fig. 2, it can be seen that strength at tensile fracture is always lower than tensile strength at any hot-pressing time, which indicated that starch/GS composite 
had certain degree of toughness. As shown in Fig. 3, tensile elongation of composite showed similar changing law with hot-pressing time as strength. When hot-pressing time was $10 \mathrm{~min}$, tensile elongation arrived at maximum value, $12.61 \%$.

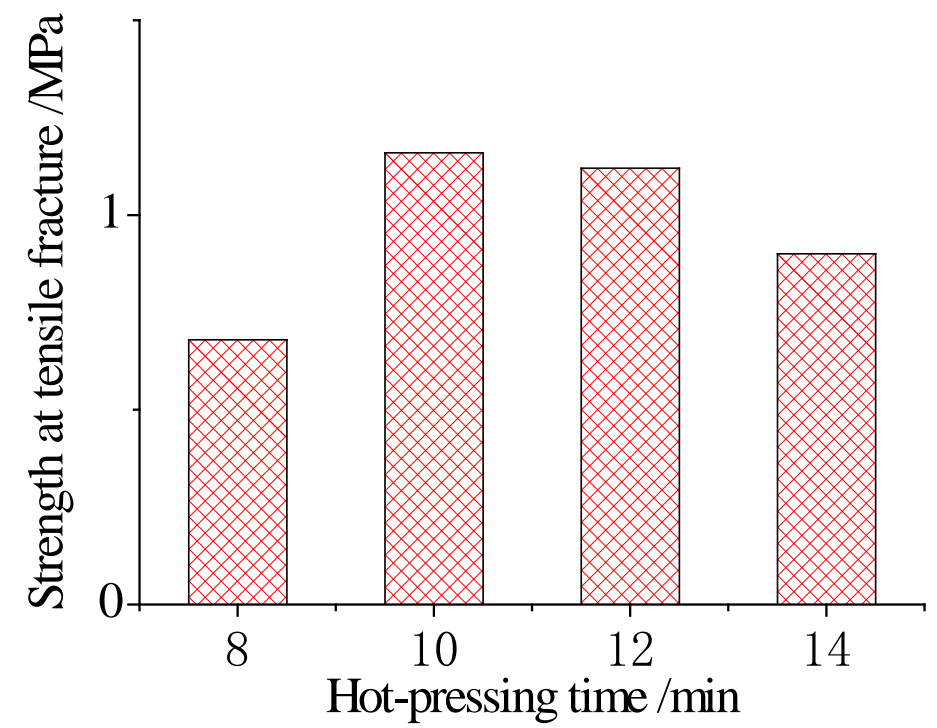

Fig. 2 The strength at tensile fracture of composites at different hot-pressing time.

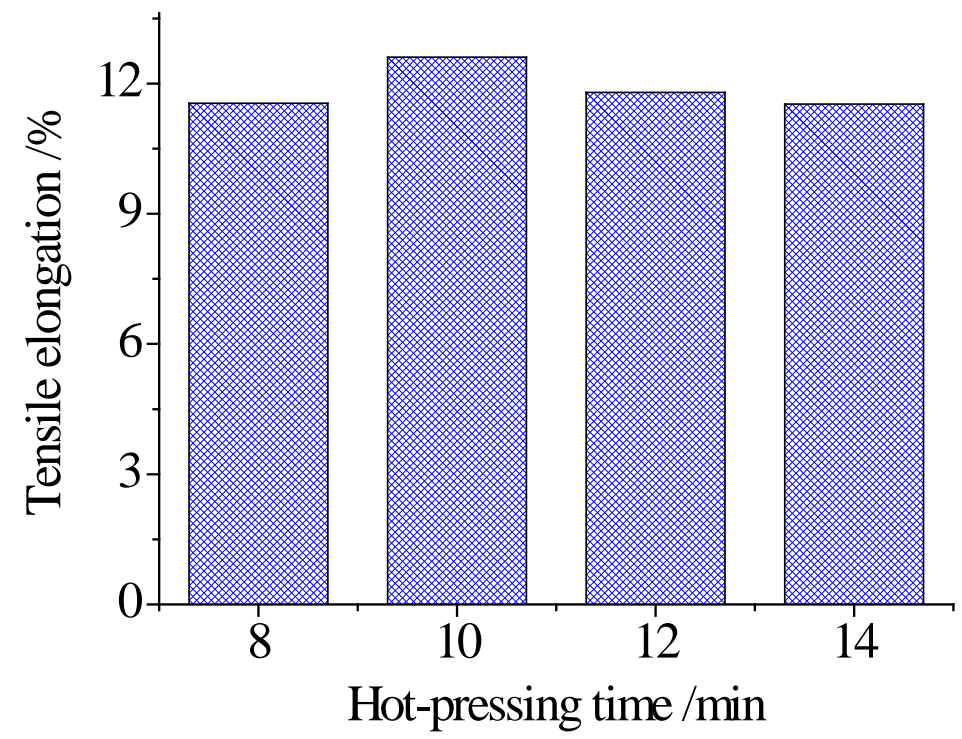

Fig. 3 The tensile elongation of composites at different hot-pressing time.

The Effect of Amount of Mixing medium on the Performance of Composite Materials. A series of starch/GS composites containing $15 \mathrm{~g}$ GS were prepared at different mixing medium amounts. Here the mixing medium was water. The change of strength with the increase of mixing medium amount was drawn in Fig. 4. It can be seen that too much or too less water both decreased tensile strength and strength at tensile fracture. In the preparation course of starch/GS composite, water was mixing medium. Otherwise, water played role of plasticizing starch. Starch can not play role of adhesion and its adhesive role depends on water. If water was too less, starch did not realize complete plasticization, fluxion and binding GS. If water was too much, starch achieved complete plasticization but composite contained more left water after hot-pressing. When water amount was 10 
$\mathrm{mL}$, tensile strength and strength at tensile fracture both reached maximum and they were 3.01 MPa and 2.83 $\mathrm{MPa}$.

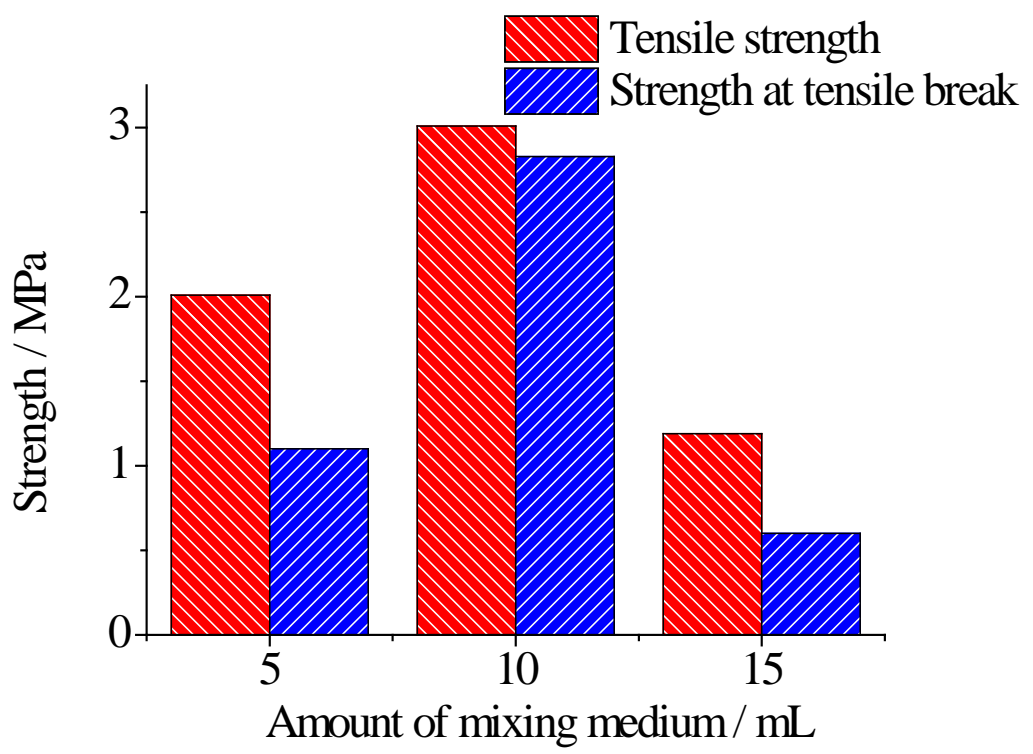

Fig. 4 The strength of composites at different amounts of mixing medium.

\section{Summary}

Performance of starch/GS composite was affected by processing conditions including hot-pressing time and amount of mixing medium. Tensile strength, strength at tensile fracture and tensile elongation first increase and then decrease with starch content. Too much or too less water both decreased tensile strength and strength at tensile fracture.

\section{Acknowledgements}

This work was financially supported by National Natural Science Foundation of China (21164002).

\section{References}

[1] Y. Kurokochi, M. Sato: Industr. Crop. Prod. Vol. 69(2015), p.55.

[2] C.B. Wu, Y. Bo: Appl. Mechan. Mater. Vol. 525(2014), p.166.

[3] N. Said, T. Bishara, A. García-Maraver and M. Zamorano: Waste Manage. Vol. 33(2013), p. 2250.

[4] J.J. Liu, C.J. Jia and C.X. He: AASRI Procedia Vol. 3(2012), p. 83.

[5] L.J. Qin, J.H. Qiu, M.Z. Liu, S.L. Ding, L. Shao, S.Y. Lü, G.H. Zhang, Y. Zhao, X. Fu: Chem. Eng. J. Vol. 166(2011), p. 772.

[6] Y. Zhao, J.H. Qiu, H.X. Feng, M. Zhang, L. Lei, X.L. Wu: Chem. Eng. J. Vol. 173(2011), p. 659.

[7] L. Zhang, Y.C. Hu: Mat. Design. Vol. 55(2014), P.19. 